\title{
Impactos dos fundos setoriais nas empresas*
}

Bruno César Araújo

Diretoria de Estudos e Políticas Setoriais de Inovação, Regulação e Infraestrutura (Diset) do Instituto de Pesquisa Econômica Aplicada (Ipea)

\section{Donald Pianto}

Departamento de Estatística da Universidade de Brasília (EST/UnB)

\section{Fernanda De Negri}

Diretoria de Estudos e Políticas Setoriais de Inovação, Regulação e Infraestrutura (Diset) do Instituto de Pesquisa Econômica Aplicada (Ipea)

\section{Luiz Ricardo Cavalcante}

Diretoria de Estudos e Políticas Setoriais de Inovação, Regulação e Infraestrutura (Diset) do Instituto de Pesquisa Econômica Aplicada (Ipea)

\section{Patrick Franco Alves}

Diretoria de Estudos e Políticas Setoriais de Inovação, Regulação e Infraestrutura (Diset) do Instituto de Pesquisa Econômica Aplicada (Ipea)

* Os autores agradecem a Erick Damasceno e a Gustavo Alvarenga pelo auxílio com os bancos de dados. Agradecem ainda as sugestões enviadas pelos pareceres anônimos. Os erros e omissões são de inteira responsabilidade dos autores. 


\section{RESUMO}

Os fundos setoriais foram instituídos no final da década de 1990, com o propósito de criar condições mais estáveis de financiamento público às atividades de ciência, tecnologia e inovação (CT\&I) no Brasil. De maneira análoga ao que se observa com outros instrumentos de incentivo à inovação nas empresas, a expectativa é que o acesso aos fundos setoriais contribuiria para o aumento dos esforços tecnológicos e para o alcance de melhores resultados pelas empresas. O objetivo deste trabalho é, portanto, avaliar o impacto desses fundos sobre os esforços tecnológicos e sobre os resultados das empresas industriais no Brasil, no período 2001 a 2006. A base teórica para a discussão é a literatura internacional que tem, recorrentemente, analisado o efeito crowding in ou crowding out de políticas de apoio à inovação nas empresas. Esses trabalhos buscam verificar se as políticas adotadas complementam os recursos alocados nas atividades de inovação pelas empresas ou se haveria simplesmente a substituição desses últimos por recursos públicos. Neste artigo, uma técnica quasi-experimental é aplicada para comparar as empresas que acessaram os fundos setoriais com aquelas que não os acessaram, usando dados de painel que incluem informações sobre esforços tecnológicos e resultados. O grupo de controle é definido com base no algoritmo de Propensity Score Matching (PSM), visando eliminar o viés de seleção no acesso aos fundos, o que faz com que, a priori, as empresas que acessam esses recursos trilhem uma trajetória distinta daquelas que não acessam. Estimativas das diferenças percentuais das taxas de crescimento dos esforços tecnológicos indicam significativo descolamento entre os grupos de tratamento e controle e permitem que se rejeite a hipótese de crowding out. Estima-se que o diferencial na taxa de crescimento do PoTec - que corresponde à proxy para os esforços tecnológicos - seja de 6,8 p.p. no primeiro ano, 11,5 p.p. no segundo, 15,7 p.p. no terceiro e 26,7 p.p. no quarto ano após o acesso. Os fundos setoriais apresentam ainda impacto positivo e significativo no pessoal ocupado total, embora apenas um impacto marginalmente significante nas exportações de alto conteúdo tecnológico tenha sido observado após quatro anos nas empresas que compõem o grupo de tratamento. Adicionalmente, uma análise preliminar dos impactos dos diferentes instrumentos que compóem os fundos setoriais permite associar a maior parte dos impactos dos recursos à concessão de crédito em condições mais favoráveis.

PalaVras-CHAVE | Fundos setoriais; Políticas de inovação; Pesquisa e desenvolvimento; Adicionalidade.

Códigos JEL | O31. 


\title{
Impacts of the Brazilian science and technology sectoral funds on the industrial firms' $R \& D$ inputs and outputs
}

\begin{abstract}
The Brazilian science and technology sectoral funds were established at the end of the 1990s, aiming at providing more stable financial resources to science, technology, and innovation (ST\&I) activities in the country. Similarly to other instruments used to foster innovation at the firm level, the sectoral funds are expected to increase firms' technological efforts as well as their result indicators. The aim of this paper is to evaluate the impacts of these funds on the industrial firms' $R \& D$ inputs and outputs in Brazil during the period between 2001 and 2006. Several papers have discussed the additionality or crowding out effects of innovation policies that involve grants and fiscal incentives, for example. In this paper, the firms which accessed the sectoral funds are compared with the ones which did not, based upon the path followed by their indicators of technological efforts (R\&D inputs) and results (R\&D outputs). The control group was defined using a Propensity Score Matching (PSM) procedure aiming at reducing the selection bias that makes firms which accessed the funds follow a different path when compared to the ones that did not. Percentage differencein-differences indicate a significant detachment between the technological efforts of the treatment and control groups and permit the hypothesis of crowding out to be rejected. The growth differential on the PoTec variable - the proxy for technological efforts - is estimated in 6.8 p.p. in the first year, 11.5 in the second, 15.7 p.p. in the third and 26.7 p.p. in the fourth year after the access to the funds. The sectoral funds also presented a significant and positive impact on the number of employees, although only a marginally significant impact on high-tech exports was observed four years after the treatment. Additionally, a preliminary analysis of the impacts of the different instruments that form the sectoral funds suggests that most impacts observed in the technological efforts can be associated to the credit at favorable conditions.
\end{abstract}

KEYwORDS | Sectoral funds; Innovation policies; Research and development; Crowding in.

JEL-Codes | O31. 


\section{Introdução}

O objetivo deste trabalho é avaliar o impacto dos fundos setoriais sobre os esforços tecnológicos e sobre os resultados das empresas industriais no Brasil, no período 2001 a 2006. Os fundos setoriais foram instituídos no final da década de 1990, com o propósito de criar condiçôes mais estáveis de financiamento público às atividades de ciência, tecnologia e inovação (CT\&I) no Brasil. Ao vincular os recursos destinados aos fundos setoriais a um conjunto abrangente de fontes de recursos, a legislação pretendeu evitar que restrições de natureza fiscal causassem descontinuidades nas políticas de CT\&I adotadas pelo governo federal. O contexto que marcou a institucionalização dos fundos foi caracterizado, ainda, pelo crescente reconhecimento da importância de se estabelecerem incentivos ao desenvolvimento tecnológico nas empresas. Isso explica porque tais recursos têm sido um dos principais instrumentos da política nacional de CT\&I para apoiar a disseminação da inovação no setor produtivo.

De maneira análoga ao que se observa com outros instrumentos de incentivo à inovação nas empresas - como o terceiro capítulo da chamada "Lei do Bem" (Lei $n^{\circ} 11.196 / 2005$ ) e a Lei de Informática (Leis no 8.248/1991 e no 10.176/2001) -, a expectativa era de que o acesso aos fundos setoriais contribuiria para o aumento dos esforços tecnológicos e para o alcance de melhores resultados pelas empresas. Em última análise, o objetivo seria a geração de externalidades positivas para o conjunto da sociedade. Assim, em linhas gerais, o argumento subjacente aos incentivos governamentais à inovação no setor produtivo apoia-se em duas premissas fundamentais: os retornos sociais dos investimentos em pesquisa e desenvolvimento são, em geral, superiores a seus retornos privados em virtude da impossibilidade de se apropriarem plenamente seus resultados no interior das empresas; e os elevados níveis de incerteza associados às atividades de inovação inibem a captação de recursos privados externos para financiar as atividades de P\&D (ARROW, 1962; HALL, 1992 apud ASCHHOFF, 2009, p. 1).

Embora a hipótese de que o acesso aos instrumentos de políticas de inovação traria benefícios para as empresas seja razoável e amparada em uma ampla variedade de políticas públicas adotadas nos países da Organização para a Cooperação e o Desenvolvimento Econômico (OCDE), o fato é que não há, ainda, evidências robustas que a comprovem para o caso do Brasil. ${ }^{1}$ A literatura internacional sobre

1 Para um relato das políticas de apoio à inovação nas empresas adotadas nos países da OCDE, ver, por exemplo, OCDE (1999). 
o tema tem, recorrentemente, analisado o efeito crowding in ou crowding out de políticas de apoio à inovação nas empresas. ${ }^{2}$ Essencialmente, esses trabalhos buscam investigar se os recursos seriam usados de forma complementar àqueles alocados pelas empresas, ou se haveria simplesmente a substituição desses últimos por recursos públicos. Trata-se, em suma, de verificar se os recursos têm sido alocados de maneira a garantir um maior envolvimento do setor produtivo com as atividades de inovação.

Este trabalho está segmentado em mais quatro seções além desta introdução. Na segunda, discutem-se os fundamentos teóricos sobre os quais se apoia a avaliação. Basicamente, são sistematizados os métodos e resultados obtidos por autores que investigaram o impacto de políticas de incentivo à inovação sobre o setor produtivo em outros países. Com base nessa revisão bibliográfica, propõem-se, na terceira seção, os métodos empregados para avaliação dos impactos dos fundos nas empresas. Em particular, procura-se destacar os procedimentos adotados para definição dos grupos de tratamento e de controle e para evitar o viés de seleção tipicamente observado em avaliações dessa natureza. Os resultados da avaliação são discutidos na quarta seção e, na quinta, apresentam-se as principais conclusões do trabalho.

\section{Fundamentação teórica}

Ao longo dos últimos dez anos, um vasto conjunto de trabalhos sobre os impactos de políticas públicas de inovação nas empresas tem sido publicado. $\mathrm{O}$ crescimento do número de publicações pode ser creditado à disseminação de políticas voltadas para o apoio à inovação nas empresas, em oposição às ações tradicionais de suporte à produção científica. Isso, é claro, não quer dizer que não existam trabalhos que, antes do final da década de 1990, tenham se debruçado sobre esse tema.

David, Hall e Toole (2000), ao sistematizarem grande parte dos trabalhos publicados sobre o tema até o final dos anos 1990, registram que os estudos podem ser segmentados em quatro grandes blocos: estudos cross-section no nível microeconômico; dados de painel no nível microeconômico; estudos agregados ou macroeconômicos; e estudos, macro ou microeconômicos, que buscam considerar a simultaneidade dos gastos públicos e privados em pesquisa e desenvolvimento (P\&D), especialmente por meio do uso de variáveis instrumentais. Ao analisar especificamente os incentivos fiscais para as atividades de P\&D, Hall e Van Reenen (2000), por sua vez, segmentam a literatura em três blocos principais: estudos de casos e eventos;

2 Várias dessas referências são discutidas na seção 2 deste trabalho. Em particular, David, Hall e Toole (2000) sistematizam os resultados obtidos por diversos estudos publicados entre meados da década de 1960 e 2000. 
experimentos naturais usando equações de demanda de P\&D e um parâmetro shift para o crédito; e quasi-experimentos usando estimativas de elasticidades de preços.

David, Hall e Toole (2000) destacam também que Hamberg (1966) teria sido o primeiro a usar regressóes econométricas de dados de empresas individuais para verificar a relação entre os gastos públicos e privados em P\&D. Na década de 1980, Lichtenberg (1984; 1987; 1988 apud DAVID, HALL; TOOLE, 2000) publicou uma série de trabalhos sobre o tema, buscando propor modelos mais consistentes e empregar técnicas econométricas mais sofisticadas. Em que pese o avanço representado por trabalhos como esses, o fato é que, antes do final dos anos 1990, não se empregavam técnicas que visavam evitar o viés de seleção e a endogeneidade tipicamente observados quando se avaliam impactos de políticas de inovação.

Essas limitações começaram a ser superadas ao longo dos últimos dez anos, quando os trabalhos passaram a empregar técnicas estatísticas que buscavam levar em consideração que as empresas capazes de ter acesso aos instrumentos de política de inovação teriam características distintas daquelas que não possuíam essa possibilidade. Como resultado, atualmente, a maioria das análises evita comparar diretamente as empresas que tiveram e as que não tiveram acesso aos instrumentos de políticas de inovação. Conforme descrito em detalhes na terceira seção deste trabalho, os procedimentos que têm sido usados consistem, essencialmente, em identificar grupos de empresas que teriam tido chances similares de acessar os instrumentos, para, em seguida, comparar aquelas que os acessaram com aquelas que não os acessaram. Procedimentos dessa natureza, adotados por exemplo por Busom (1999), são hoje razoavelmente consensuais nas avaliações de políticas públicas de inovação realizadas desde então. De fato, uma vasta produção bibliográfica - especialmente de autores ligados ao Centre for European Economic Research $(Z E W)$ - tem buscado verificar o impacto de políticas públicas de P\&D nas empresas.

A lógica subjacente às análises assume que os atributos individuais da empresa determinam sua probabilidade de acessar os instrumentos de políticas de inovação e pressupõe, embora nem sempre de forma explícita, uma relação de causalidade entre o acesso a esses instrumentos, os esforços tecnológicos das empresas e a obtenção de algum tipo de resultado. Conforme indicado na Figura 1, as políticas de inovação envolvem instrumentos que vão desde subsídios ("grants") e incentivos fiscais e financeiros até o acesso privilegiado ao conhecimento produzido por universidades ou centros de pesquisa. Os esforços tecnológicos ( $R \& D$ inputs), por sua vez, são frequentemente aferidos por meio dos gastos empresariais em P\&D, embora, nas circunstâncias em que esses dados não estejam disponíveis, outras proxies - especial- 
mente aquelas relacionadas com a contratação de pessoal técnico-científico - possam ser usadas. Já os resultados ( $R \& D$ outputs) podem estar associados ao incremento dos resultados de inovação (por exemplo, a obtenção de patentes ou a introdução de produtos inovadores no mercado), ao desempenho da empresa (por exemplo, o aumento do faturamento ou de suas exportaçóes de alto conteúdo tecnológico) e às externalidades positivas para o conjunto da sociedade. Na prática, essa última categoria - que constitui, em última análise, a razão de ser das políticas - não é considerada nas avaliaçóes em virtude da impossibilidade de quantificá-las de forma objetiva. $^{3}$

\section{FIGURA 1}

Estrutura lógica das pesquisas sobre impactos de políticas de inovação nas empresas

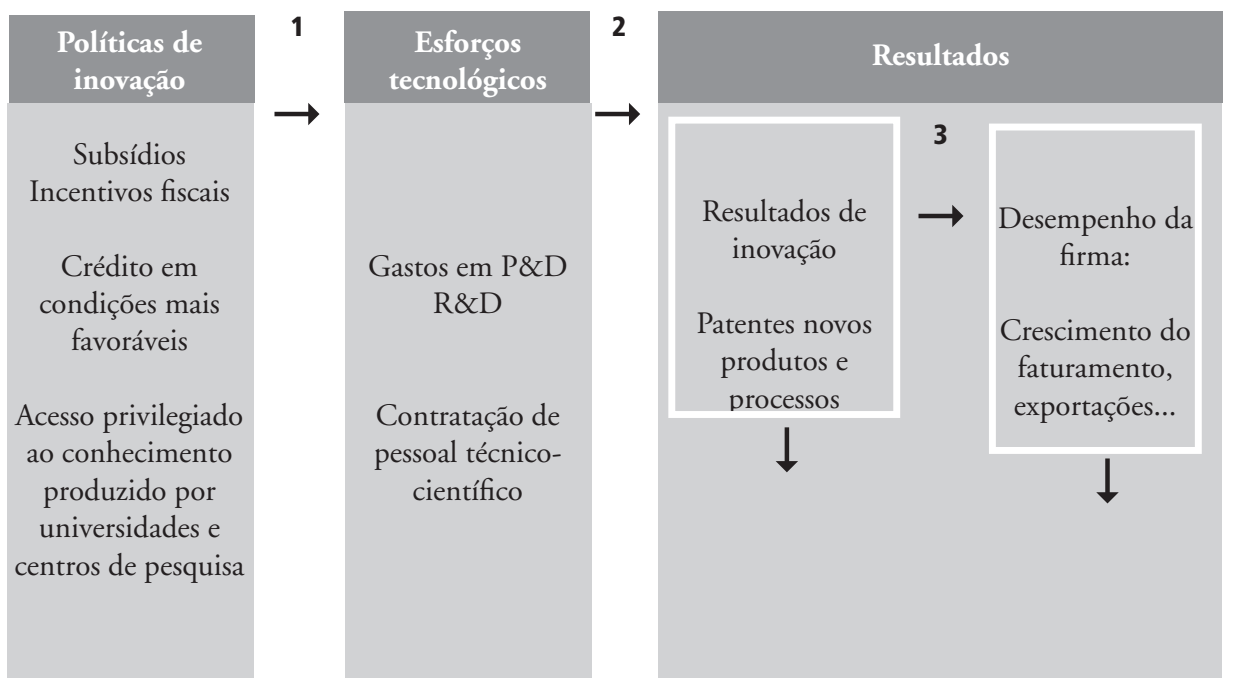

Fonte: Elaboração dos autores.

Não há dúvida de que a maioria dos trabalhos referentes ao tema concentra-se na avaliação dos impactos das políticas públicas de inovação sobre os esforços tecnológicos das empresas (a seta indicada pelo número 1 na figura). Em particular, predominam estudos de avaliação de impactos de programas de subsídios e de incentivos fiscais sobre os gastos empresariais em P\&D (por exemplo, ALMUS; CZARNITZKI, 2003). Nesses casos, não se investiga se os esforços tecnológicos produzem algum

3 Trata-se de uma limitação severa, mas especialmente incontornável e extensiva à avaliação de qualquer política pública. A impossibilidade de se avaliarem de forma quantitativa as externalidades sugere que pode haver circunstâncias em que os resultados são apropriados por outros atores sociais, e não pelas empresas; assim, se os impactos forem reduzidos para a firma isso não quer dizer, necessariamente, que a política foi mal sucedida. 
tipo de resultado, mas, apenas, se as políticas geram esforços tecnológicos (isto é, se há crowding in ou crowding out em decorrência das políticas adotadas).

Do ponto de vista metodológico, predominam as técnicas de matching não paramétrico descritas na terceira seção deste artigo. Aschhoff (2009, p. 3) menciona cerca de 20 estudos que avaliam os efeitos dos subsídios públicos à P\&D sobre os gastos privados nessas atividades. A autora destaca que a maioria dos trabalhos conclui ser possível rejeitar a hipótese de full crowding out, embora os resultados estejam longe de levar a um consenso. Um exemplo desse tipo de análise pode ser visto em Almus e Czarnitzki (2003), que analisam os impactos dos subsídios à inovação concedidos a empresas da antiga Alemanha Oriental sobre seus gastos em P\&D. Os autores usam o matching não-paramétrico para evitar o viés de seleção, controlando por vasto conjunto de variáveis que envolvem, inclusive, o setor de atuação, o tamanho da empresa (medido pelo seu pessoal ocupado), o nível de internacionalização (exportações e importações, por exemplo), a concentração setorial e a origem do capital (se proveniente da então Alemanha Oriental, da Alemanha Ocidental ou de outro país). A principal conclusão é que o acesso aos subsídios aumenta os esforços tecnológicos das empresas em cerca de 4\%. Um exercício similar, para o caso brasileiro, foi publicado por Avellar (2008), que avaliou os impactos do Programa de Desenvolvimento Tecnológico Industrial (PDTI), concluindo que o acesso ao programa leva as empresas beneficiárias a dobrarem seus investimentos em $\mathrm{P} \& \mathrm{D}$.

A produção teórica sobre o tema não tem se limitado a tratar do possível efeito crowding out, uma vez que alguns trabalhos buscam avaliar a efetividade das políticas públicas de inovação, levando em conta o custo a elas associado. Uma relevante questão levantada pela literatura é se um aumento de uma unidade monetária nos recursos públicos destinados ao financiamento das atividades de P\&D levaria a uma elevação mais do que proporcional nos gastos privados. De forma geral, prevalece, nesse tipo de trabalho, a regra "one-to-one": uma unidade monetária de recursos públicos induz uma unidade monetária equivalente em $\mathrm{P} \& \mathrm{D}$ por parte das empresas. ${ }^{4}$ Contudo, a análise de efetividade não requer a adoção dessa premissa, tendo em vista as externalidades positivas que se associam às atividades de $\mathrm{P} \& \mathrm{D}$ (MOHNEN; LOKSHIN, 2009).

Alguns trabalhos estendem a análise descrita anteriormente aos resultados associados ao desempenho inovador da empresa, alcançando a seta número 2 na Figura 1. Aschhoff (2009), por exemplo, verifica os impactos de subsídios governamentais nos inputs e outputs das atividades de P\&D nas empresas na Alemanha. Em par-

4 Este tipo de análise por vezes é referido na literatura por "bang-for-the-buck". 
ticular, os resultados são aferidos com base em patentes e na introdução de novos produtos no mercado. Trabalhos como esse, entretanto, não avaliam os resultados mensurados com base no desempenho das empresas (crescimento do faturamento, da produtividade e da rentabilidade e exportações, por exemplo). Em outras palavras, os trabalhos enfatizam os impactos das políticas públicas sobre aquilo que, na Figura 1, denominou-se de "resultados de inovação".

Um conjunto de dificuldades metodológicas impede, em geral, que as análises alcancem o desempenho da empresa, quer ele seja atribuído aos esforços tecnológicos, quer ele o seja às atividades de inovação (seta 3 na Figura 1). Em primeiro lugar, a relação entre esforços tecnológicos, inovação e desempenho não é necessariamente linear, e a sequência dos eventos pode levar a resultados distintos. Em segundo lugar, o caráter path dependent dessa relação não pode ser capturado, nos modelos econométricos, simplesmente por meio do uso de dummies temporais. Além disso, os prazos requeridos para que os esforços tecnológicos e as atividades de inovação convertam-se em desempenhos superiores podem variar bastante em função da própria natureza do processo de inovação, sendo que os riscos inerentes às atividades de P\&D fazem com que um percentual relativamente elevado dos projetos não obtenha, de fato, resultados expressivos.

Essas dificuldades não impediram que Crépon, Duguet e Mairesse (1998) modelassem tais impactos em três estágios:

- Estágio 1 - decisão de investir em inovação;

- Estágio 2 - resultados do investimento em inovação;

- Estágio 3 - impactos da inovação sobre a produtividade.

Esses três estágios estão contidos na área envolta pela linha tracejada da Figura 1. Os principais resultados convergem com os fatos estilizados mais comuns na literatura sobre inovação (por exemplo, os autores reafirmam que a probabilidade de as empresas engajarem-se em atividades de P\&D cresce com o porte e o marketshare). Em particular, com relação aos resultados, Crépon, Duguet e Mairesse (1998) concluem que: os resultados de inovação, aferidos com base em patentes e na comercialização de produtos inovadores, são positivamente correlacionados com os esforços de pesquisa; e a produtividade e os resultados de inovação estão positivamente correlacionados, mesmo após se controlar pela qualificação da mão de obra e pela intensidade de capital físico. Da mesma forma, Kannebley, Sekkel e Araújo (2010) mostram que a inovação está positivamente relacionada ao crescimento do pessoal ocupado, da receita líquida de vendas, da produtividade do trabalho e do capital e market share, especialmente quando ocorre simultaneamente em produto 
e em processo. Em resumo, os resultados obtidos por Crépon, Duguet e Mairesse (1998) e por Kannebley, Sekkel e Araújo (2010) indicam que, em que pesem as dificuldades metodológicas para se associar o desempenho das empresas a seus esforços tecnológicos, é possível esperar uma correlação positiva entre essas duas dimensões. Uma análise dos impactos de políticas públicas de inovação sobre os esforços tecnológicos (seta 1), as atividades de inovação (seta 2) e o desempenho da empresa (seta 3) foi realizada por De Negri, De Negri e Lemos (2008a). Os autores avaliam os impactos do Programa de Apoio ao Desenvolvimento Tecnológico da Empresa Nacional (ADTEN) sobre os gastos em P\&D, patentes e o desempenho econômico e financeiro das empresas. As conclusões indicam a existência de: evidências robustas de que o ADTEN influencia positivamente os gastos em P\&D; sinais de impactos positivos sobre o número de pedidos de patentes, embora os coeficientes obtidos não sejam significativos; iii) há impactos positivos e significativos na receita líquida de vendas e no pessoal ocupado; e impactos positivos, porém não significativos, na produtividade. Esses mesmos autores, em um esforço similar, avaliam os impactos do Fundo Nacional para o Desenvolvimento Científico e Tecnológico (DE NEGRI; DE NEGRI; LEMOS, 2008b). As conclusões são bastante similares às do exercício envolvendo o ADTEN.

\section{Procedimentos metodológicos}

Do ponto de vista metodológico, a avaliação dos impactos dos fundos setoriais nas empresas apoia-se na comparação dos indicadores de esforços tecnológicos e resultados das firmas que acessaram os fundos (grupo de tratamento) com os indicadores daquelas que não os acessaram (grupo de controle). Esse procedimento foi aplicado em três etapas que definem a estrutura desta seção do trabalho. Assim, descrevem-se, na subseção 3.1, as variáveis usadas no modelo e, na subseção 3.2, os procedimentos de construção da base de dados e dos grupos de tratamento e controle. Finalmente, na subseção 3.3, discutem-se os procedimentos econométricos empregados para comparar as amostras obtidas.

\subsection{Variáveis}

Empregado uma base original formada por cerca de 13 mil projetos apoiados pelos fundos setoriais, definiu-se, para cada empresa que se beneficiou dos recursos, uma variável de "estreia" correspondente ao primeiro ano de acesso. Pretendeu-se, com 
isso, evitar que empresas que já viessem sendo apoiadas pelos fundos por vários anos fossem diretamente comparadas com aquelas que recebiam pela primeira vez os recursos. Assim, o impacto dos fundos setoriais foi avaliado a partir do momento em que as empresas acessam esse instrumento.

Uma variável binária para o acesso aos fundos setoriais foi usada para definir o grupo de tratamento. Essa variável assume o valor 1 no ano de estreia, 0 no período anterior e missing no período posterior.

$\mathrm{O}$ acesso aos fundos setoriais pode envolver: projetos cooperativos com universidades e centros de pesquisa, tanto na condição de executante como na condição de interveniente; crédito em condições mais favoráveis; ${ }^{5}$ e subvenções ${ }^{6}$.

Neste trabalho, privilegiou-se a análise dos impactos dos fundos setoriais em geral, não tendo sido estabelecida distinção entre as três modalidades indicadas ou o acúmulo de duas ou mais modalidades de apoio pela mesma empresa. Implicitamente, assume-se que, ao se engajarem como intervenientes em projetos cooperativos, as empresas beneficiam-se de transbordamentos das atividades de P\&D desenvolvidas por universidades e centros de pesquisa. Embora essa modalidade de apoio seja obviamente menos direta do que crédito ou subvenção, assume-se não haver distinção entre seus impactos esperados. ${ }^{7}$ Além disso, a magnitude do apoio recebido não foi considerada na análise.

Conforme indicado anteriormente, os esforços tecnológicos são tradicionalmente aferidos com base nos gastos em P\&D interno e externo. No Brasil, esses gastos são medidos por meio da Pesquisa de Inovação Tecnológica (Pintec), realizada a cada três anos. A periodicidade trienal da Pintec e o fato de que a última edição disponível quando este trabalho foi elaborado refere-se a 2005 impedem o uso dos gastos em $\mathrm{P} \& \mathrm{D}$ como indicador de esforços tecnológicos das empresas que obtiverem o apoio dos fundos, uma vez que, em grande parte dos casos, o acesso aos instrumentos ocorreu entre 2005 e 2006 e seus impactos não teriam sido capturados pela edição da pesquisa aqui empregada. Diante dessa limitação, optou-se por utilizar uma variável

5 No caso dos projetos reembolsáveis (crédito), ainda que os recursos não tenham origem diretamente nos Fundos Setoriais, as empresas foram incluídas no grupo de tratamento em virtude da equalização de taxas de juros, cujo funding provém, necessariamente, dos fundos.

6 Não houve, no período, empresas que tiveram acesso à subvenção econômica, prevista pela Lei de Inovação (Lei n 10.973/2004) e que corresponde ao mais novo instrumento de incentivo à inovação oferecido pela Finep. A variável foi incluída para permitir análises futuras.

7 Essa opção decorre, essencialmente, do reduzido número de observações na amostra, particularmente no caso do crédito e da subvenção. Entretanto, é preciso reconhecer que os projetos cooperativos podem incentivar, em alguns casos, a transferência de atividades de pesquisa para as universidades e centros de pesquisa (o que, princípio, poderia motivar uma redução do PoTec). Por essa razão, análises econométricas preliminares foram realizadas visando distinguir os impactos de cada instrumento (ver seção 4). 
intitulada "pessoal ocupado técnico-científico" (PoTec), que corresponde à soma dos valores associados aos seguintes grupos ocupacionais da Classificação Brasileira de Ocupações (CBO): pesquisadores, engenheiros, diretores e gerentes de $\mathrm{P} \& \mathrm{D}$ e o que chamamos de "profissionais científicos" (Quadro 1). A opção pelo uso dessa proxy segue ainda a proposição pioneira de Blank e Stigler (1957).

QUADRO 1

Grupos ocupacionais do PoTec

\begin{tabular}{l|lc}
\hline \multicolumn{1}{c}{ Grupos ocupacionais } & \multicolumn{1}{c}{ Profissionais } & Código CBO(1) \\
\hline Pesquisadores & Pesquisadores & 203 \\
& Engenheiros mecatrônicos & 222 \\
\hline \multirow{2}{*}{ Engenheiros } & Engenheiros agrônomos e de pesca & 214 \\
\hline \multirow{2}{*}{ Diretores e gerentes de P\&D } & Engenheiros civis, etc. & 1237 \\
& Diretores de P\&D & 1426 \\
& Gerentes de P\&D & 201 \\
& Biotecnologistas, geneticistas, pesquisadores & \\
\hline & em metrologia e especialistas em calibrações & 211 \\
& metereológicas & 212 \\
\hline Profissionais "científicos" & Matemáticos, estatísticos e afins & 213 \\
& Profissionais de informática & 221 \\
\hline & Físicos, químicos e afins & \\
\hline
\end{tabular}

Fonte: Araújo, Cavalcante e Alves (2009).

(1) Os códigos de três dígitos são referentes aos subgrupos da $\mathrm{CBO}$ e os de quatro dígitos às famílias.

Originalmente proposto por Gusso (2006) e ajustado, em seguida, por Araújo, Cavalcante e Alves (2009), o PoTec, conforme assinalam esses últimos autores, apresentou, em 2005, um coeficiente de correlação com os gastos internos e externos em P\&D superior a 90\%. Dessa forma, há evidências robustas de que o PoTec é uma proxy adequada dos esforços tecnológicos. Como o PoTec pode ser calculado com base nos dados da Relação Anual de Informaçôes Sociais (Rais), é possível acompanhar sua evolução anual durante o período considerado na análise.

Finalmente, os indicadores de resultados foram segmentados em indicadores de resultados de inovação e indicadores de desempenho da firma. Em particular, as taxas de crescimento da empresa (aferidas por meio da evolução de seu pessoal ocupado total) e as exportaçôes de alto conteúdo tecnológico foram empregadas neste trabalho. Rigorosamente, o crescimento seria mais adequadamente aferido por meio da evolução do faturamento. A ausência desse dado, entretanto, impôs o uso 
do pessoal ocupado como proxy do tamanho da empresa. Embora esse indicador apresente limitaçóes evidentes (por exemplo, nas circunstâncias em que as atividades de inovação acarretam elevação da produtividade de mão de obra), assume-se aqui que, na média, há uma correlação positiva entre crescimento do faturamento e aumento do pessoal ocupado total. Com relação às exportaçōes de alto conteúdo tecnológico, empregaram-se dados do Ministério do Desenvolvimento, Indústria e Comércio Exterior (MDIC) tabulados de acordo com a classificação da OCDE.

\subsection{Base de dados e procedimentos de amostragem}

A base de dados que fundamentou a análise aqui apresentada corresponde a um painel de empresas no período de 2000 a 2007. Considerando-se os instrumentos mencionados na subseção anterior, mais de mil empresas acessaram os fundos no período até 2008. Neste trabalho, foram consideradas, tanto no grupo de tratamento como no de controle, apenas as empresas industriais presentes na Rais em 2006 e 2007 e que tivessem mais de cinco funcionários. Nesses dois anos, vigorava a versão 2.0 da Classificação Nacional de Atividades Econômicas (CNAE 2.0), que foi definida como padrão da classificação setorial das empresas analisadas neste trabalho. ${ }^{8}$ Com isso, a amostra de empresas tratadas reduziu-se a cerca de 600 observaçôes". Além disso, para avaliar o impacto dos Fundos Setoriais nas empresas é necessário um período de defasagem. Por essa razão, e em virtude da disponibilidade de informaçōes de outras bases, o grupo de tratamento incluiu apenas as empresas apoiadas pelos fundos entre 2001 e 2006, o que acarretou mais uma redução na amostra. Após a aplicação desses filtros, o grupo de tratamento foi formado por um total de 344 empresas, conforme indicado na Tabela 1.

9 De acordo com o segundo relatório do convênio MCT/Finep/Ipea/UFMG para avaliação dos fundos setoriais (DE NEGRI et al., 2009). Convém observar que este trabalho usa uma amostra de 13.433 projetos apoiados pelos fundos setoriais entre 2000 e 2008. Atualmente, o Ministério da Ciência e Tecnologia (MCT) já possui informações de cerca de 20 mil projetos apoiados pelos fundos. Mesmo para o subconjunto analisado neste trabalho, não há informações sobre as empresas apoiadas por fundações estaduais de amparo à pesquisa no âmbito do Programa de Apoio à Pesquisa em Empresas (Pappe Subvenção) e/ou pelo Serviço Brasileiro de Apoio às Micro e Pequenas Empresas (Sebrae), que correspondem a uma parcela significativa das empresas apoiadas pelos fundos. Também não foram incorporadas à amostra usada neste trabalho as empresas apoiadas pelo Projeto Inovar (capital de risco) e as que receberam apoio para contratação de pesquisadores via bolsas do Programa de Capacitação de Recursos Humanos para Atividades Estratégicas (RHAE). Estima-se que, em todas essas modalidades, os fundos setoriais tenham apoiado cerca de 2 mil empresas no período analisado. 
TABELA 1

Estreia das empresas nas diferentes modalidades de apoio dos fundos setoriais 2001-2006

\begin{tabular}{c|c|c|c|c}
\hline Anos & $\begin{array}{c}\text { Somente } \\
\text { projetos } \\
\text { cooperativos }\end{array}$ & $\begin{array}{c}\text { Somente } \\
\text { crédito }\end{array}$ & Ambos & Total \\
\hline 2001 & 0 & 17 & 0 & 17 \\
2002 & 47 & 13 & 3 & 63 \\
2003 & 13 & 5 & 0 & 18 \\
2004 & 79 & 0 & 0 & 79 \\
2005 & 56 & 25 & 2 & 83 \\
2006 & 48 & 32 & 4 & 84 \\
\hline
\end{tabular}

Fonte: Finep/MCT/Rais (MTE). Elaboração dos autores.

Conforme indicado na Tabela 1, a maior parte do grupo de tratamento é formada por empresas que se envolveram apenas em projetos cooperativos (cerca de $70 \%$ do total). Como mencionado anteriormente, nenhuma empresa teve acesso à subvenção no intervalo considerado, dado que o instrumento foi operacionalizado pela Finep somente no período mais recente.

Uma vez definido o grupo de tratamento, o grupo de controle foi obtido por meio de um algoritmo de Propensity Score Matching (PSM), visando eliminar o viés de seleção no acesso aos fundos, o que faz com que, a priori, as empresas que acessam esses recursos trilhem uma trajetória distinta daquelas que não acessam. ${ }^{10}$ Esse procedimento de controle foi aplicado para que as análises pudessem capturar plenamente os impactos dos fundos setoriais. A hipótese fundamental, nesse caso, é de que o acesso aos fundos é condicionado por variáveis observáveis (hipótese de "seleção sobre observáveis"). O PSM permite a correção do viés de seleção ao emparelhar empresas tratadas e não tratadas que compartilham características observáveis. Essas características - usualmente relativas ao período anterior ao analisado, a fim de evitar causalidade reversa, ou seja, de a própria trajetória dos indicadores voltar a influenciar a probabilidade do acesso aos instrumentos de apoio à inovação - são usadas para calcular a probabilidade de acesso aos fundos $p\left(x_{i}\right)=\operatorname{Pr}\left(S F_{i, t}=1 \mid x_{i, t-1}\right)$.

Assim, a aplicação do procedimento segue a ordem: características observáveis determinam a probabilidade de acesso aos fundos; empresas com características semelhantes e iguais probabilidades (do ponto de vista estatístico) de acesso aos fundos têm suas trajetórias comparadas; calculam-se os diferenciais das trajetórias 10 Ver Wooldridge (2002, p. 603-644). 
das variáveis de interesse entre empresas que acessaram os fundos e aquelas que não o fizeram. Portanto, na prática, a trajetória seguida pelos esforços tecnológicos ou pelos resultados é calculada de maneira condicional à probabilidade de tratamento, permitindo uma comparação não viesada. ${ }^{11}$

Neste trabalho, a estimativa de $p\left(x_{i}\right)$ foi feita com base em um modelo probit, usando as seguintes séries de características observáveis das empresas:

- $\quad$ PoTec (defasado e considerando sua interação com dummies de ano);

- pessoal ocupado total (defasado e considerando sua interação com dummies de ano); ${ }^{12}$

- variáveis binárias para empresa multinacional (dummy que assume o valor 1 para empresas para as quais a participação do capital estrangeiro é superior a 50\%) e para sociedades anônimas;

- setor;

- região.

O modelo adotado e as variáveis escolhidas para caracterizar as empresas são consistentes com os procedimentos empregados na maioria dos estudos mencionados na segunda seção deste trabalho.

Uma vez que o acesso aos fundos setoriais é um evento raro (o que pode prejudicar o ajuste de modelos probabilísticos), um processo de amostragem repetida (bootstrap) com amostras diferentes (porém similares) para os grupos de tratamento e de controle foi aplicado no cálculo dos escores de propensão. Esse tipo de procedimento é tipicamente utilizado em análises de escores de crédito, por exemplo, nas quais a inadimplência é também um evento raro. Após o processo de amostragem repetida, a probabilidade predita da empresa $i$ pertencente ao grupo de tratamento acessar os fundos foi denominada $p_{i}{ }_{i}$, ao passo que $p_{j}^{0}$ corresponde à probabilidade predita da empresa $j$ que não teve acesso aos recursos.

Além do processo de amostragem repetida, foi empregado um corte de suporte comum, de modo que as empresas que acessaram os fundos e tinham probabilidade reduzida de fazê-lo (de tal forma que não havia empresas não tratadas com probabilidades similares) foram excluídas da amostra. Inversamente, as empresas que acessaram os fundos e tinham uma elevada probabilidade de fazê-lo (de modo que não se pôde identificar contrafactuais em sua vizinhança de probabilidade) foram também excluídas da amostra. Isso foi feito considerando-se a probabilidade

11 Essa é a condição de independência da participação no tratamento e o resultado para o grupo não tratado, isto é, $y^{0} \perp S F \mid x_{i}$. O método PSM se baseia nessa suposição.

12 Essa variável, na prática, corresponde ao porte da empresa. 
mínima para os casos e a probabilidade máxima para os controles como limites, de modo que o balanceamento foi aplicado somente para as empresas cujos escores de propensão pertenciam à interseção dos grupos de tratamento e controle.

Finalmente, após a obtenção dos grupos de tratamento e de controle, o balanceamento das amostras foi verificado, visando garantir que não houvesse diferenças significativas entre os dois grupos remanescentes.

\subsection{Procedimentos de comparação}

Para comparar a trajetória seguida pelas empresas que acessaram os fundos setoriais com a trajetória daquelas que não os acessaram, ajustaram-se as equações indicadas a seguir:

$$
y_{i, t+n}=\alpha_{t+n}+\beta S F_{i, t}+\varepsilon_{i, t+n}
$$

ou, em sua versão em primeira diferença:

$$
\Delta y_{i, t+n}=\alpha_{t+n}^{\prime}+\beta^{\prime} S F_{i, t}+\varepsilon_{i, t+n}
$$

Nessas equações:

$S F_{i, t}$ : indicador de acesso da empresa $i$ aos fundos setoriais no momento $t$. Conforme indicado na subseção 3.1, essa dummy pode assumir o valor 0 (se a empresa não teve acesso aos fundos setoriais) ou 1 (caso contrário). Empresas que estrearam antes de $t$ ou entre $t+1 \mathrm{e} t+n$ foram excluídas dessas estimativas.

$y_{i, t+}$ : indicador de esforço tecnológico ou de resultado da empresa $i$ no momento $t+n$, com $n \geq-1$.

$\Delta y_{i, t+n}$ : primeira diferença da variável $y$ para a empresa $i$, ou seja, $y_{i, t+n}-y_{i, t+n-1}$ com $n \geq 1$ e $y_{i, t-} y_{i, t-1}$ se $n=0$.

A equação (1) representa simplesmente o teste de médias nas variáveis de interesse entre as empresas que acessaram os fundos setoriais e as que não acessaram. A diferença em $y$ entre as duas categorias de firmas, em $n$ períodos à frente do tratamento, é expressa pelo coeficiente $\beta$, e a significância estatística dessa diferença é dada pela significância desse coeficiente. Por seu turno, o coeficiente $\alpha_{t+n}$ representa a média da variável de interesse $y$ para o grupo dos não tratados.

A equação (2) visa captar a diferença-das-diferenças (DID) entre os dois grupos de empresas - isto é, o quanto a mais a variável de interesse cresceu no grupo de 
tratamento vis-à-vis o grupo de controle. Estes métodos complementam a análise em nível, fornecendo uma interpretação bastante interessante, sobretudo quando os grupos de tratamento e controle já são diferentes antes do tratamento. Além disso, são úteis para eliminar diferenças entre casos e controles invariantes no tempo, mesmo que não observáveis. Assim, $\alpha$ 'é $\Delta \bar{y}^{0}$ e o $\beta$ ' neste caso representa a estimativa DID de $\left(\Delta y^{-1}-\Delta_{y}^{-0}\right)$, em que os sobrescritos 1 e 0 indicam, respectivamente, se as empresas tiveram acesso aos fundos ou não.

Foi estimada ainda uma versão da equação (2) em sua forma logarítmica:

$$
\Delta \log y_{i, t+n}=\alpha_{t+n}^{\prime}+\beta^{\prime \prime} S F_{i, t}+\varepsilon_{i, t+n}
$$

Onde,

$$
\begin{aligned}
& \exp \left(\alpha^{\prime \prime}\right)=\frac{\bar{y}_{t+n}^{0}}{\bar{y}_{t+n-1}^{0}} \\
& \beta^{\prime \prime}=\left(\log \bar{y}_{t+n}^{1}-\log \bar{y}_{t+n-1}\right)-\left(\log \bar{y}_{t+n}^{0}-\log \bar{y}_{t+n-1}^{0}\right)=\log \left(\frac{\bar{y}_{t+n}^{1}}{\overline{y_{t+n-1}}}\right)-\log \left(\frac{\bar{y}_{t+n}^{0}}{\bar{y}_{t+n-1}}\right)
\end{aligned}
$$

Ou, rearranjando,

$$
\exp \left(\beta^{\prime \prime}\right)=\frac{\frac{\bar{y}_{t+n}}{\overline{-1}}}{\frac{y_{t+n-1}}{-y_{t+n}}} \frac{\bar{y}_{t+n-1}}{y^{01}}
$$

Assim, se $\exp (\alpha ”)>1$, pode-se concluir que houve crescimento da variável entre os períodos $t+n$ e $t+n-1$ no grupo de controle. Por sua vez, se $\exp \left(\beta^{\prime \prime}\right)>1$, então o crescimento relativo da variável de interesse foi maior para o grupo de tratamento do que no de controle. Neste trabalho, optou-se por exibir uma transformação de $\beta$, na forma $\left[\exp \left(\beta^{\prime}\right)-1\right] \times 100$, que mostra o diferencial da taxa de crescimento relativo da variável de interesse no grupo de controle, em termos percentuais.

Desse modo, no primeiro conjunto, intitulado "empresas em geral", o grupo de controle corresponde ao conjunto das empresas industriais com total de pessoal ocupado superior a cinco. Nesse caso, espera-se um maior descolamento entre os grupos de tratamento e de controle ao longo do tempo, uma vez que não se considera o viés de seleção que faz com que, em princípio, as empresas com acesso aos fundos setoriais trilhem uma trajetória distinta daquelas que não acessaram. 
Conforme destacado na seção 3.2, uma melhor aproximação do real efeito causal do acesso aos fundos setoriais pode ser obtida quando se comparam grupos de empresas similares, isto é, cujas probabilidades de acesso aos recursos são similares, exceto pelo fato de que aquelas pertencentes ao grupo de tratamento efetivamente os acessaram, enquanto as do grupo de controle não os acessaram.

Pode-se assumir, portanto, que o efeito causal do acesso aos fundos setoriais na empresa $i$ no momento $t+n$ é definido como $y_{i, t+n}^{1}-y_{i, t+n}^{0}$. O problema fundamental de inferência causal nesse caso é que $y_{i, t+n}^{0}$ não é observado para as empresas que tiveram acesso aos fundos setoriais (isto é, para aquelas para as quais se observa $\left.y_{i, t+n}^{1}\right)$. Diante dessa constatação, nada poderia ser dito sobre o impacto causal do acesso aos fundos setoriais sem alguma hipótese sobre o valor de $y_{i, t+n}^{0}$. Este valor hipotético - chamado de contrafactual - é obtido a partir de uma inferência baseada na comparação de um resultado factual (observado) com um resultado contrafactual. Trata-se, assim, daquilo que, na literatura econométrica, é denominado avaliação de tratamento. Em particular, Wooldridge (2002) define o efeito médio do tratamento sobre o tratado $(A T T)$ por:

$$
A T T=E\left(y_{i, t+s}^{1}-y_{i, t+s}^{0} \mid F S_{i, t}=1\right)=E\left(y_{i, t+s}^{1} \mid F S_{i, t}=1\right)-E\left(y_{i, t+s}^{0} \mid F S_{i, t}=1\right)
$$

Para contornar o fato de que $E\left(y_{i, t+n}^{0} \mid F S_{i, t}=1\right.$ não é observável, constrói-se um grupo de controle, estimando-se uma função do tipo $E\left(y_{i, t+n}^{0} \mid F S_{i, t}=0\right)$. O resultado médio do grupo que não teve acesso aos fundos setoriais identifica o resultado médio contrafactual para o grupo que os acessou.

No entanto, o acesso aos fundos setoriais não é um evento aleatório; e supõe-se que ele esteja condicionado pelas características da empresa. Dessa forma, atribuir simplesmente $E\left(y_{i, t+n}^{0} \mid F S_{i, t}=1\right)=E\left(y_{i, t+n}^{0} \mid F S_{i, t}=0\right)$ e calcular diretamente as diferenças de médias em $y$ entre quem acessou ou não os fundos setoriais poderia viesar os resultados da análise. A endogeneidade da escolha pode advir do fato de que o acesso aos fundos setoriais correlaciona-se a características das empresas, configurando então um problema de seletividade da amostra. Esse problema é contornado usando os escores de propensão descritos na seção 3.2, de modo que uma expressão geral para o estimador $A T T_{p s m}$ é dada por:

$$
A T T_{p s m}=\frac{1}{N} \sum_{i \in F S=1}\left(y_{i}^{1}-\sum_{j \in C\left(x_{i}\right)} w(.) y_{j}^{0}\right)
$$


Onde $C\left(x_{i}\right)$ identifica os controles para a empresa $i$. Na equação acima, 0 $<w() \leq 1,. \operatorname{sum}(w())=$.1 e $w($.$) é uma função que atribui pesos à j-ésima$ empresa correspondente ao contrafactual da firma que teve acesso aos fundos setoriais e $N$ é o número de empresas que acessaram e que tiveram contrafactuais. Neste trabalho, um algoritmo de PSM kernel foi empregado no cálculo dos pesos $w($.$) .$

\section{Resultados}

Os procedimentos indicados na seção anterior foram aplicados a uma amostra originalmente formada por 344 empresas tratadas e cerca de 113 mil empresas não tratadas. Escores de propensão que representam a probabilidade de uma firma acessar os instrumentos de apoio da Finep foram calculados após 450 repetições do probit com amostras formadas por 330 empresas para o grupo de tratamento e 2 mil para o de controle. $\mathrm{O}$ suporte comum explica porque o grupo de tratamento foi reduzido para 330 empresas. Os resultados obtidos para o pseudo- $\mathrm{R}^{2}$ (cerca de $50 \%$ ) sugerem um ajuste apropriado para o emparelhamento das empresas. Além disso, conforme indicado na Tabela 2, o ajuste obtido para a maioria das variáveis pode ser considerado satisfatório.

\section{TABELA 2}

Distribuição dos coeficientes probit após 450 repetições

\begin{tabular}{lc|cc}
\hline \multicolumn{1}{c|}{ Variáveis } & Estimativa (Mediana) & \multicolumn{2}{c}{$\begin{array}{c}\text { [Intervalo de conf. } \\
\text { de 90\%] }\end{array}$} \\
\hline $\begin{array}{l}\text { Log do pessoal técnico-científico } \\
\text { ocupado }\left(\mathrm{t}_{-1}\right)\end{array}$ & 0,528 & 0,342 & 0,755 \\
Log do pessoal ocupado total $\left(\mathrm{t}_{-1}\right)$ & 0,208 & 0,326 & 0,441 \\
Multinacional (dummy) & $-0,933$ & $-1,53$ & $-0,431$ \\
Sociedade anônima (dummy) & 0,549 & 0,032 & 1,24 \\
Pseudo R2 & 0,495 & 0,449 & 0,539 \\
Log verossimilhança & $-738,7$ & $-797,4$ & $-673,4$ \\
\hline
\end{tabular}

Fonte: Elaboração dos autores.

Nota: Dummies setoriais, dummies de ano e de interação não foram exibidas na tabela.

Conforme mostrado na Tabela 2, a maioria dos coeficientes é significativamente diferente de zero. Basicamente, o modelo probabilístico indica que empresas que 
já empregam pessoal técnico-científico têm maiores probabilidades de acesso aos fundos. Da mesma forma, aquelas de maior porte também têm maiores probabilidades de acesso. Empresas nacionais possuem maiores chances do que multinacionais (conforme indica o coeficiente negativo e significante obtido para a dummy multinacional). A um nível de $10 \%$ de confiança, o modelo é inconclusivo quanto ao fato de que sociedades anônimas teriam maiores chances de acesso aos recursos (uma vez que o intervalo de confiança para o quinto percentil inclui o valor zero). Independentemente disso, pode-se considerar que o ajuste do modelo é bastante satisfatório. Essa percepção é reforçada ao se verificar que a mediana obtida para o pseudo-R $\mathrm{R}^{2}$ foi de 0,495 .

Os escores de propensão obtidos após os procedimentos descritos anteriormente permitiram a construção de grupos de tratamento e controle com características similares no momento precedente ao tratamento. A Tabela 3 mostra as características dos grupos de tratamento e controle em condições de não emparelhamento e de emparelhamento no ano anterior ao acesso aos fundos setoriais.

TABELA 3

Esforços tecnológicos e resultados em t-1

\begin{tabular}{|c|c|c|c|c|}
\hline \multirow{2}{*}{ Variáveis } & \multicolumn{2}{|c|}{ Não emparelhadas } & \multicolumn{2}{|c|}{ Emparelhadas } \\
\hline & Tratamento & Controle & Tratamento & Controle \\
\hline $\begin{array}{l}\text { Pessoal técnico- } \\
\text { científico empregado }\end{array}$ & 46,09 & 0,42 & 21,97 & 26,84 \\
\hline Pessoal ocupado total & 998 & 48 & 737 & 1,023 \\
\hline $\begin{array}{l}\text { Exportações de alto } \\
\text { conteúdo tecnológico } \\
\text { (US\$ mil) }\end{array}$ & 3.367 & 64 & 3.229 & 3.836 \\
\hline
\end{tabular}

Fonte: Elaboraçāo dos autores.

As condições de balanceamento foram satisfeitas, uma vez que os grupos de tratamento e controle exibiram os mesmos valores médios para as variáveis usadas no probit em $t_{-1}$. Além disso, os dois grupos têm os mesmos valores médios para as variáveis de interesse, uma vez que os valores indicados nas colunas referentes a "emparelhadas" não são significativamente (do ponto de vista estatístico) diferentes. Alcançar a condição de balanceamento é interessante porque indica que o viés de seleção foi eliminado e que as empresas que compóem o grupo de controle têm características similares às daquelas do grupo de tratamento após a aplicação dos pesos encontrados com o procedimento de kernel matching. 
Uma vez obtidos os grupos de tratamento e controle diretamente comparáveis, a trajetória seguida por cada amostra foi comparada conforme indicado na Tabela 4, para cada variável de interesse: a média do grupo de controle; diferencial em nível dos grupos de tratamento e controle; diferença-das-diferenças em nível dos grupos de tratamento e controle; e diferença percentual das taxas de crescimento entre os grupos de tratamento e controle.

TABELA 4

Esforços tecnológicos e resultados entre t-1 e $t+4$

\begin{tabular}{|c|c|c|c|c|c|c|c|}
\hline Va & iáveis & $t_{-1}$ & $t_{0}$ & $t_{1}$ & $t_{2}$ & $t_{3}$ & $t_{4}$ \\
\hline \multirow{4}{*}{$\begin{array}{l}\text { Esforços } \\
\text { tecnológicos } \\
\text { (pessoal } \\
\text { técnico- } \\
\text { científico } \\
\text { ocupado } \\
\text { PoTec) }\end{array}$} & $\begin{array}{l}\text { Média do grupo } \\
\text { de controle }\end{array}$ & 26.84 & 27.65 & 28.08 & 32.25 & 32.77 & 34.10 \\
\hline & $\begin{array}{l}\text { Diferencial em } \\
\text { nível dos grupos } \\
\text { de tratamento e } \\
\text { controle }\end{array}$ & $-4,87^{\text {ns }}$ & $-3,14^{\text {ns }}$ & $-2,34^{\mathrm{ns}}$ & $-4,58^{\text {ns }}$ & $-9,62^{* \star *}$ & $-9,03^{n s}$ \\
\hline & $\begin{array}{l}\text { Diferença-das- } \\
\text { diferenças em } \\
\text { nível dos grupos } \\
\text { de tratamento e } \\
\text { controle }\end{array}$ & - & $1,69^{*}$ & $1,30^{*}$ & $1,75^{\mathrm{ns}}$ & $2,18^{\mathrm{ns}}$ & $6,22^{*}$ \\
\hline & $\begin{array}{l}\text { Diferença } \\
\text { percentual } \\
\text { das taxas de } \\
\text { crescimento } \\
\text { entre os grupos } \\
\text { de tratamento e } \\
\text { controle }\end{array}$ & - & $5,47^{\star \star}$ & $6,82^{* * \star}$ & $11,52^{* \star \star}$ & $15,72^{* * *}$ & $26,74^{* *}$ \\
\hline \multirow{4}{*}{$\begin{array}{l}\text { Tamanho } \\
\text { da empresa } \\
\text { (pessoal } \\
\text { ocupado } \\
\text { total) }\end{array}$} & $\begin{array}{l}\text { Média do grupo } \\
\text { de controle }\end{array}$ & $1.022,75$ & $1.082,50$ & $1.127,11$ & $1.249,71$ & $1.244,12$ & $1.545,86$ \\
\hline & $\begin{array}{l}\text { Diferencial em } \\
\text { nível dos grupos } \\
\text { de tratamento e } \\
\text { controle }\end{array}$ & $-285,33^{* * *}$ & $-287,71^{* \star *}$ & $-265,70^{* * *}$ & $-392,07^{* \star *}$ & $-348,07^{*}$ & $-364,96^{\text {ns }}$ \\
\hline & $\begin{array}{l}\text { Diferença-das- } \\
\text { diferenças em } \\
\text { nível dos grupos } \\
\text { de tratamento e } \\
\text { controle }\end{array}$ & - & $-2,03^{\text {ns }}$ & $28,83^{\text {ns }}$ & $30,43^{\text {ns }}$ & $-21,93^{\text {ns }}$ & $-30,91^{\mathrm{ns}}$ \\
\hline & $\begin{array}{l}\text { Diferença } \\
\text { percentual } \\
\text { das taxas de } \\
\text { crescimento } \\
\text { entre os grupos } \\
\text { de tratamento e } \\
\text { controle }\end{array}$ & - & $1,82^{\text {ns }}$ & $6,82^{* * *}$ & $9,64^{* * *}$ & $11,52^{*}$ & $16,07^{\text {ns }}$ \\
\hline
\end{tabular}


TABELA 4

Esforços tecnológicos e resultados entre $t-1$ e $t+4$

\begin{tabular}{|c|c|c|c|c|c|c|c|}
\hline \multicolumn{2}{|c|}{ Variáveis } & $t_{-1}$ & $t_{0}$ & $t_{1}$ & $t_{2}$ & $t_{3}$ & $t_{4}$ \\
\hline \multirow{4}{*}{$\begin{array}{l}\text { Exportações } \\
\text { de alto } \\
\text { conteúdo } \\
\text { tecnológico } \\
\text { (US\$ mil) }\end{array}$} & $\begin{array}{l}\text { Média do grupo } \\
\text { de controle }\end{array}$ & $3.836,37$ & $4.597,01$ & $5.489,91$ & $6.377,65$ & $6.646,15$ & $8.405,20$ \\
\hline & $\begin{array}{l}\text { Diferencial em } \\
\text { nível dos grupos } \\
\text { de tratamento e } \\
\text { controle }\end{array}$ & $-607,01^{\mathrm{ns}}$ & $18,00^{\text {ns }}$ & $-1.811,10^{\text {ns }}$ & $-1.737,93^{\text {ns }}$ & $681,28^{\text {ns }}$ & $-4.304,97^{*}$ \\
\hline & $\begin{array}{l}\text { Diferença-das- } \\
\text { diferenças em } \\
\text { nível dos grupos } \\
\text { de tratamento e } \\
\text { controle }\end{array}$ & - & $625,70^{\text {ns }}$ & $-190,14^{\mathrm{ns}}$ & $10,28^{\text {ns }}$ & $1.122,26^{\mathrm{ns}}$ & $-210,31^{\text {ns }}$ \\
\hline & $\begin{array}{l}\text { Diferença } \\
\text { percentual } \\
\text { das taxas de } \\
\text { crescimento } \\
\text { entre os grupos } \\
\text { de tratamento e } \\
\text { controle }\end{array}$ & - & $12,52^{\text {ns }}$ & $6,18^{\mathrm{ns}}$ & $15,03^{\text {ns }}$ & $14,22^{\text {ns }}$ & $35,80^{*}$ \\
\hline
\end{tabular}

Fonte: Elaboração dos autores.

Nota: ns: não significante; ${ }^{*}$ significante a $10 \%{ }^{* *}$ significante a $5 \%{ }^{* * *}$ significante a $1 \%$.

Com relação aos esforços tecnológicos, a Tabela 4 mostra que a diferença percentual das taxas de crescimento entre os grupos de tratamento e controle é positiva e significante (a 1\% ou 5\%) durante todo o período de análise. Em outras palavras, as taxas de crescimento do PoTec das empresas que acessaram os fundos são significativamente superiores às daquelas que não os acessaram. Com efeito, após quatro anos, o grupo de tratamento acumulou uma taxa de crescimento mais de 25 pontos percentuais superior à do grupo de controle. Essa conclusão permanece válida mesmo ao se considerar que os resultados das diferenças-dasdiferenças em nível nem sempre são significantes. Esse aparente paradoxo pode ser explicado com base nos efeitos de escala: o grupo de controle apresentou maiores níveis de PoTec (conforme mostra o sinal negativo do diferencial em nível). Entretanto, ao se subtrair a diferença inicial, a diferença-das-diferenças em nível jamais é negativa e significante para nenhum dos resultados e é positiva e significante para a maior parte do horizonte de tratamento. Uma vez que as empresas do grupo de tratamento têm menores níveis de PoTec, acrescentar a mesma quantidade de pessoal do que os controles implica uma maior taxa de crescimento. Em resumo, as empresas do grupo de tratamento conseguem crescer em nível no mesmo ritmo que as empresas (maiores) do grupo de controle. Esses 
resultados confirmam o impacto positivo e significante dos fundos setoriais sobre os esforços tecnológicos das empresas.

Os fundos setoriais também tiveram impactos positivos sobre o desempenho das empresas aferido por pessoal ocupado total. De fato, impactos significativos sobre taxas de crescimento no primeiro e no segundo anos após o acesso aos fundos setoriais puderam ser observados (a diferença percentual das taxas de crescimento entre os grupos de tratamento e controle em $t_{+1}$ e $t_{+2}$ são positivas e significantes a $1 \%)$. Entretanto, apenas um impacto marginalmente significante nas exportações de alto conteúdo tecnológico foi observado após quatro anos nas empresas que compõem o grupo de tratamento (diferença percentual das taxas de crescimento $\mathrm{em} \mathrm{t}_{+4}$ ). Uma possível explicação para esse resultado pode envolver os requisitos de competitividade internacional não capturados pelo modelo. Em outras palavras, ainda que as empresas consigam crescer no mercado interno, os requisitos para competir no mercado externo são mais severos e podem estar associados a um esforço tecnológico ainda maior. Outra possível explicação é que o impacto sobre as exportações de alto conteúdo tecnológico pode requerer a estreia no mercado internacional, o que demandaria mais tempo para ser percebido do que as séries temporais usadas neste trabalho são capazes de capturar. Isso explicaria porque somente após quatro anos observou-se um efeito marginalmente significante.

Finalmente, foi realizada uma análise preliminar dos impactos dos diferentes instrumentos que compóem os fundos setoriais (projetos cooperativos, crédito e subsídios) sobre o pessoal ocupado técnico-científico. Nesse caso, regrediu-se a primeira diferença do logaritmo do PoTec entre $t_{+1}$ to $t_{+4}$ ) contra variáveis binárias correspondentes à modalidade de tratamento acessada (projetos cooperativos, crédito e a interação desses dois tratamento, uma vez que não houve acesso a subsídios nos período analisado). Os pesos na regressão foram idênticos aos obtidos anteriormente usando o algoritmo de kernel matching. Conforme indicado na seção 3, projetos cooperativos podem ser considerados uma forma menos direta de apoio do que créditos ou subsídios, uma vez que, em geral, não implicam a transferência direta de recursos do governo para as empresas, mas apenas os transbordamentos das atividades de pesquisa conduzidas em universidades ou centros de pesquisa. Por outro lado, créditos e subsídios que, de alguma forma, pressupõem a transferência de recursos do governo para as empresas tenderiam a apresentar impactos maiores. Embora essa análise pareça um tanto prematura em função das amostras reduzidas, alguns resultados preliminares estão registrados na Tabela 5, que mostra a diferença percentual das taxas de crescimento do PoTec 
entre os grupos de tratamento e controle para os projetos cooperativos, os créditos e a interação entre esses dois instrumentos.

TABELA 5

Diferença percentual das taxas de crescimento do PoTec para cada instrumento

\begin{tabular}{lcc|c|c|c}
\hline \multicolumn{1}{c}{ Instrumentos } & $\mathbf{t}_{\mathbf{1}}$ & $\mathbf{t}_{\mathbf{2}}$ & $\mathbf{t}_{\mathbf{3}}$ & $\mathbf{t}_{\mathbf{4}}$ \\
\hline \multirow{2}{*}{ Projetos cooperativos } & 0,0366 & 0,0887 & 0,0933 & 0,0571 \\
& $(1,31)$ & $(2,21)$ & $(1,52)$ & $(0,48)$ \\
\multirow{2}{*}{ Créditos } & 0,1587 & 0,2152 & 0,3890 & 0,5787 \\
& $(2,83)$ & $(1,98)$ & $(2,41)$ & $(3,78)$ \\
Interação & $-0,2544$ & $-0,6257$ & $-0,7790$ & $-0,9764$ \\
& $(-0,97)$ & $(-2,22)$ & $(-1,56)$ & $(-2,36)$ \\
\hline
\end{tabular}

Fonte: Elaboração dos autores.

Nota: Estatística t indicada abaixo dos coeficientes.

Ao se confrontarem as estatísticas $\mathrm{t}$ indicadas abaixo dos coeficientes na Tabela 5 , fica evidente que os projetos cooperativos mostram impactos menos significativos (ou simplesmente não tiveram impacto algum) do que os créditos. Esse resultado preliminar sugere que os projetos cooperativos têm menor impacto sobre o PoTec das empresas. ${ }^{13}$ Os dados da Tabela 5 indicam ainda que os impactos dos fundos podem ser amplificados à medida que modalidades de apoio mais diretas se disseminarem.

\section{Conclusões}

Neste trabalho avaliou-se o impacto dos fundos setoriais sobre esforços tecnológicos e resultados das empresas industriais no Brasil, no período entre 2001 e 2006. Para isso, compararam-se, para as empresas que acessaram os fundos setoriais e para aquelas que não os acessaram, as trajetórias de três variáveis principais: esforços tecnológicos (pessoal técnico-científico ocupado); tamanho da empresa (cuja proxy foi o pessoal ocupado total); e exportações de alto conteúdo tecnológico. O grupo de controle foi definido com base no algoritmo de Propensity Score Matching (PSM), visando eliminar o viés de seleção no acesso aos fundos, o que faz com que, a priori, as empresas que acessam esses recursos trilhem uma trajetória distinta daquelas que não acessam. 13 De fato, conforme mostrado na Tabela 5, exceto em t2 os impactos sobre as diferença-das-diferenças não são
estatisticamente significativos para os projetos cooperativos. 
Com relação aos esforços tecnológicos, estimativas das diferenças percentuais das taxas de crescimento do pessoal técnico-científico ocupado indicam um significativo descolamento entre os grupos de tratamento e controle. Com efeito, a taxa de crescimento do PoTec das empresas que acessaram os fundos são significativamente maiores do que as daquelas que não acessaram os recursos. Estima-se que o diferencial na taxa de crescimento do PoTec - que corresponde à proxy para os esforços tecnológicos - entre as empresas que acessaram os fundos em comparação àquelas de características semelhantes no que tange à probabilidade de acesso seja de 6,8 p.p. no primeiro ano, 11,5 p.p. no segundo, 15,7 p.p. no terceiro e 26,7 p.p. no quarto ano após o acesso.

Esses resultados permitem que se rejeite a hipótese de crowding out. Quando os resultados são aferidos por meio do pessoal ocupado total, os impactos dos fundos setoriais também foram positivos e significantes no primeiro e segundo anos após o acesso aos recursos. Por outro lado, apenas um impacto marginalmente significante nas exportações de alto conteúdo tecnológico foi observado após quatro anos nas empresas que compõem o grupo de tratamento. Uma análise preliminar dos impactos dos diferentes instrumentos que compõem os fundos setoriais permite associar a maior parte dos impactos dos recursos à concessão de crédito em condições mais favoráveis.

Trabalhos futuros podem aprofundar as análises aqui apresentadas de duas formas básicas. Em primeiro lugar, pode-se avaliar com maior precisão os impactos dos diferentes instrumentos que compóem os fundos separadamente, em especial do recente instrumento subvenção econômica. Análises dessa natureza podem ajudar os formuladores de políticas a ajustar os instrumentos de apoio a objetivos específicos (por exemplo, o apoio a empresas emergentes de pequeno porte ou a atividades de $\mathrm{P} \& \mathrm{D}$ de empresas de grande porte pode requerer diferentes instrumentos). Em segundo lugar, a magnitude do apoio recebido pode ser considerada na análise, uma vez que se espera que os impactos dos fundos tendem a ser maiores à medida que aumenta o volume de recursos destinados a cada projeto.

\section{Referências bibliográficas}

ALMUS, M.; CZARNITZKI, D. The effects of public R\&D subsidies on firms' innovation activities: the case of Eastern Germany. Journal of Business and Economic Statistics, v. 21, n. 2, p. 226-236, 2003. 
ARAÚJO, B. C.; CAVALCANTE, L. R.; ALVES, P. Variáveis proxy para os gastos empresariais em inovação com base no pessoal ocupado técnico-científico disponível na Relação Anual de Informações Sociais (RAIS). Radar: Tecnologia, Produção e Comércio Exterior, n. 5, p. 16-21, 2009.

ASCHHOFF, B. The effect of subsidies on R\&D investment and success: do subsidy history and size matter? Centre for European Economic Research, 2009 (Discussion paper, n. 09-032).

AVELLAR, A. P. Avaliação do impacto do PDTI sobre o gasto em atividades de inovação em P\&D das empresas industriais. In: DE NEGRI, J. A.; KUBOTA, L. C. (Orgs.). Politicas de incentivo à inovação tecnológica no Brasil. Brasília: Ipea, 2008.

BLANK, D. M.; STIGLER, G. J. The demand and supply of scientific personnel. New York: National Bureau of Economic Research, 1957.

BUSOM, I. An empirical evaluation of the effects of R\&D subsidies. Berkeley: University of California, May 1999 (Burch working paper, n. B99-05).

CRÉPON, B.; DUGUET, E.; MAIRESSE, J. Research, innovation, and productivity: an econometric analysis at the firm level. NBER, Aug. 1998 (Working paper, 6696).

DAVID, P. A.; HALL, B. H.; TOOLE, A. A. Is public R\&D a complement or substitute for private R\&D? A review of the econometric evidence. Research Policy, v. 29, p. 497-529, 2000.

DE NEGRI, J. A.; DE NEGRI, F.; LEMOS. M. B. O impacto do programa ADTEN sobre o desempenho e o esforço tecnológico das empresas industriais brasileiras. In: DE NEGRI, J. A.; KUBOTA, L. C. (Orgs.). Políticas de incentivo à inovação tecnológica no Brasil. Brasília: Ipea, 2008a.

. O impacto do FNDCT sobre o desempenho e o esforço tecnológico das empresas industriais brasileiras. In: DE NEGRI, J. A.; KUBOTA, L. C. (Orgs.). Politicas de incentivo à inovação tecnológica no Brasil. Brasília: Ipea, 2008b.

DE NEGRI, F; ALVES, P.; KUBOTA, L. C.; CAVALCANTE, L. R; DAMASCENO, E. C. Perfil das empresas integradas ao sistema federal de C,T\&I no Brasil e aos fundos setoriais: uma análise exploratória. Brasília: Ipea, nov. 2009. Mimeografado.

GUSSO, D. Agentes da inovação: quem os forma, quem os emprega? In: DE NEGRI, J. A.; DE NEGRI, F.; COELHO, D. (Orgs.). Tecnologia, exportação e emprego. Brasília: Ipea, 2006.

HALL, B.; VAN REENEN, J. How effective are fiscal incentives for R\&D? A review of the evidence. Research Policy, v. 29, p. 449-469, 2000.

KANNEBLEY JR., S.; SEKKEL, J. V.; ARAÚJO, B. C. Economic performance of Brazilian manufacturing firms: a counterfactual analysis of innovation impacts. Small Business Economics, p. 1-15, 2008. 
MOHNEN, P.; LOKSHIN, B. What does it take for an R\&D tax incentive policy to be effective? United Nations University, Maastricht Economic and Social Research and Training Centre on Innovation and Technology, 2009 (Working paper series, 014).

OCDE. Organização para a Cooperação e o Desenvolvimento Econômico. Managing national innovation systems. Paris: OECD, 1999.

WOOLDRIDGE, J. M. Econometric analysis of cross section and panel data. Cambridge (MA): The MIT Press, 2002.

ENDEREÇO PARA CORRESPONDENCIA:

Bruno César Araújo - bruno.araujo@ipea.gov.br

SBS Qd. 1, BI. J, Ed. BNDES - 11 ${ }^{\circ}$ andar

Brasília, DF. CEP: 70.076-900

Donald Pianto - dpianto@gmail.com

Universidade de Brasília

Instituto de Ciências Exatas - Departamento de Estatística

Campus Universitário Darcy Ribeiro - Asa Norte

Brasília, DF. CEP: 70910-900

Fernanda De Negri-fernanda.denegri@mct.gov.br

SQN 212, Bloco A, ap. 605

Brasília, DF. CEP: 70864-010

Luiz Ricardo Cavalcante - ricardo.cavalcante@ipea.gov.br

SBS Qd. 1, BI. J, Ed. BNDES - $11^{\circ}$ andar

Brasília, DF. CEP: 70.076-900

Patrick Franco Alves - patrick.alves@ipea.gov.br

SBS Qd. 1, BI. J, Ed. BNDES - $11^{\circ}$ andar

Brasília, DF. CEP: 70.076-900 
